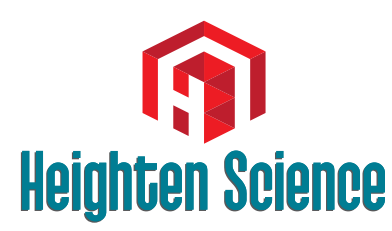

P U B L I C I T I O N S Corporation
*Address for Correspondence: Lucio Mango, Nuclear Medicine Department, S.CamilloForlanini, General Hospital, Rome, Italy, Email: I.mango@scf.gov.it

Submitted: 19 December 2016

Approved: 20 January 2017

Published: 22 February 2017

Copyright: @ 2017 Mango L. This is an open access article distributed under the Creative Commons Attribution License, which permits unrestricted use, distribution, and reproduction in any medium, provided the original work is properly cited

Check for updates
Editorial

\section{Theranostics: A Unique Concept to Nuclear Medicine}

\author{
Lucio Mango* \\ Nuclear Medicine Department, S.Camillo-Forlanini General Hospital, Rome, Italy
}

\section{EDITORIAL}

Nuclear Medicine is an integral part of modern healthcare. The use of radioactive nuclides tagged biomolecules, evaluating their distribution in human bodies by SPECT or PET systems, provides longitudinal sets of volumetric and quantitative images that can be used to diagnose a wide range of disease and/or assess response to disease specific treatments [1].

Nuclear Medicine therapy, also before known as radio-metabolic, consists in replacing cited diagnostic nuclides with alternative radionuclides that emit a "curative" type of radiations such as beta- or alpha-particles [2].

The eventual presence of a contemporary gamma emission by the nuclide utilized also allows the execution of scintigraphic investigations after its administration, useful for monitoring the distribution of the radio-pharmaceutical in the body and make the most of the time a predictive estimation of the dose received from the target compared to the administered activity [3].

Treatment with radioactive isotopes has been the first clinical application of Nuclear Medicine, when, in the early '40s, the Phosphorus-32 was used for polycythemia and some forms of leukemia [4,5] and subsequently the administration of iodine-131 was adopted for the therapy of thyroid disease $[4,6]$. Than nuclear medicine therapy has come a long and successful road, with the synthesis of many classes of radiopharmaceuticals, from those receptorial [5] to monoclonal antibodies [7,8], to various molecular probes.

Nuclear medicine treatment presents a very low risk, for example, to cause the onset of cancer in treated subjects, so that often these therapies are also used for benign diseases. Around 3 years ago, more than one hundred nuclear therapy research trials were in progress internationally, particularly in the United States [9]. Nuclear medicine physicians have practiced this form of combined diagnostic-therapeutic procedures for several decades, exploiting known thyroid-selective properties of radioiodine or using small molecule analogs of norepinephrine or engineered biologics, such as peptides, specific for somatostatin receptor [10].

Calculation of the radiation amount absorbed by body tissues is critical for the success of the treatment $[11,12]$. The objective is to estimate the dose to be administered to the patient as to provide maximum therapeutic effect but at the same time limit excessive irradiation of healthy tissues (especially the bone marrow and kidneys).

As mentioned before can be used a radio-pharmaceutical labelled by a radionuclide with emission of therapeutic radiation such as beta or alpha particles and contemporary gamma emission. This option is not always achieved, but more often it is possible to 
utilize molecules labelled with different nuclides, gamma rays emitter for diagnosis purpose and the beta- or alpha- particles emitter for treatment. Beta-emitting radioisotopes are classified as low LET and kill cells indirectly, primarily by ionizing reactive oxygen species that cause single-stranded DNA damage.

Alpha particles are the heaviest that are ejected from the nucleus of specific radioactive isotopes and can cause catastrophic damage to the cells in their path. They travel a short linear distance (a few cell diameters) from the decaying nucleus and deposit a very large amount of energy in the path they travel [13].

Authors of many groups have shown the power of molecular targeted $\alpha$-particle therapy in various preclinical and clinical settings. For example, Wild et al. directly compared the efficacy and toxicity of a bombesin peptide radiolabeled with either an $\alpha$-particle-emitter $\left({ }^{213} \mathrm{Bi}\right)$ or a $\beta$ particle emitter $\left({ }^{177} \mathrm{Lu}\right)$ in a preclinical mouse xenograph model of prostate cancer. They reported that the $\alpha$-particle therapy had a $100 \%$ response rate $(70 \%$ complete and $30 \%$ partial), compared with a $30 \%$ response rate $(20 \%$ complete and $10 \%$ partial) for $\beta$ particle emitter therapy, showing the power of using high-LET molecular targeted therapy [14].

Several study protocols have used radionuclide therapy as an adjuvant to externalbeam radiation therapy. Exposure to high-dose-rate external-beam irradiation might be expected to alter the uptake of bone-seeking radiopharmceuticals in the irradiated site, depending on the precise sequence of treament delivery [15]. However response rates are consistently high and treatment is well tolerated, even in heavily pretreated patients [15].

This concept, known as theranostics (therapy-diagnostics) consists in a combination between administration of a biomolecule labelled by a gamma emitter radionuclide for diagnostic scintigraphic purpose and subsequent administration of the same molecule labelled by a radionuclide beta-or alpha-emitter for therapeutic purposes. From an historical perspective, the concept underlying the theranostic strategy are well known in nuclear medicine and constituted the basis of many nuclear imaging procedures currently used in clinical practice [16]. One method for converting a purely imaging probe into a single-entity theranostic agent is by simply switching the radionuclide from a $\gamma$-emitter $\left({ }^{111} \mathrm{In}\right)$ to a $\beta$-emitter $\left({ }^{90} \mathrm{Y}\right)$.

Radiolabeled peptides, for example, have been an important class of compounds in radio-pharmaceutical sciences and nuclear medicine for more than 20 years. Despite strong research efforts, only somatostatin-based radio-peptides have a real impact on patient care, both diagnostically and therapeutically. [ ${ }^{111}$ In-diethylenetriaminepentaacetic acid ${ }^{0}$ ]-octreotide is commercially available for imaging. Imaging was highly improved by the introduction of PET radionuclides such as ${ }^{68} \mathrm{Ga},{ }^{64} \mathrm{Cu}$, and ${ }^{18} \mathrm{~F}$. Two peptides are successfully used in targeted radionuclide therapy when bound to somatostatin and labeled with ${ }^{90} \mathrm{Y}$ and ${ }^{177} \mathrm{Lu}$ [17].

Not only the gamma-emitting radionuclides are used in diagnostics; this is well known in nuclear medicine as long employs positronic nuclides for a better delineation and classification of the tumor mass by means of PET-CT equipment. Currently practiced molecular imaging in the form of PET with the ${ }^{18}$ F-labeled glucose analog, FDG, has revolutionized medicine, most significantly in oncology. The role of FDG PET, now synonymous with molecular imaging, has become an integral part of patient management for cancer staging, restaging, and monitoring therapy response for a great number of indications [16].

Current nuclear medicine diagnostic techniques include non-invasive Positron Emission Tomography (PET) and Single Photon Emission Computed Tomography (SPECT) utilizing, respectively, positron and gamma emitting radionuclides for the 
generation of the signal. Both external and internal radiotherapy can be directed by diagnostic PET and SPECT, and diagnostic imaging and radiotherapy are merging into theranostics resulting in more personalized medicine [18].

Most of positronic tracers must be produced by cyclotrons. Except for 18-Fluorine, it is necessary to have the production site close to nuclear medicine departments. Generator-derived radionuclides for PET/CT imaging are promising for optimizing targeted radiotherapy by an individual patient-based approach, applying pretherapeutic evaluation, as well as dosimetric calculations, and for measuring treatment response after radionuclide therapy [19].

In fact radionuclide generator systems provide an alternative route to access medically relevant radionuclides. The ${ }^{99} \mathrm{Mo} /{ }^{99 \mathrm{~m}} \mathrm{Tc}$ system, since decades being the prototype of a "medical" radionuclide generator, is still the basic source of diagnostic radiopharmaceuticals, today covering approximately $80 \%$ of all nuclear medical procedures worldwide.

In addition to the ${ }^{99} \mathrm{Mo} /{ }^{99 \mathrm{~m}} \mathrm{Tc}$ generator, with its daughter nuclide emitting low energetic photon radiation dedicated to SPECT imaging and scintigraphy [20], recent interest has focused on analogue generator systems with potential for molecular imaging using PET, such as the ${ }^{68} \mathrm{Ge} /{ }^{68} \mathrm{Ga}$ and ${ }^{82} \mathrm{Sr} /{ }^{82} \mathrm{Rb}$ generators. So that, in the context of generator-based positron-emitting radionuclides the concept of theranostics is also true.

Theranostics can leverage nuclear medicine strengths to develop platforms for identifying new biologic targets, predict possible off-target effects, and provide practical means for objective and quantifiable criteria as endpoints in monitoring therapy response. Drugs of most chemical or even inorganic compositions that are designed to be specific for a cellular or biochemical target may be rendered into an imaging agent by the appropriate conjugation with imaging radionuclides that can be detected. This key concept also underscores the value of the latest generation of potentially transformative biomedical materials that are scaled at the nanometer level [10]. Nanoparticles represent an ideally suited theranostic platform predominantly because of their modular construction.

On a purely experimental basis the frontiers of theranostics can turn a therapeutic aid to an imaging agent through appropriate and complex manipulation and labeling with the proper radionuclides. This has demonstrated by some authors who created a positronic prostate tracer from a therapeutic agent [21]. The goal of patient-specific activity prescription offers the only realistic prospect of enhancing response rates within predictable toxicity limits and of progressing beyond symptom palliation to earlier intervention for survival gain [15].

\section{REFERENCES}

1. Hacker $M$, Beyer $T$, Baum RP, Kalemis A, Lammertsma AA, et al. Nuclear medicine innovations help (drive) healthcare (benefits). Eur J Nucl Med Mol Imaging. 2015; 42: 173-175. Ref.: https://goo.gl/vAgYqG

2. Mango L, Pacilio M. Therapy with Alpha Rays. ARC Journal of Radiology and Medical Imaging. 2016; 1:1-3.

3. Pacilio M, Ventroni G, Basile C, lalongo P, Becci D, et al. Improving the dose-myelotoxicity correlation in radiometabolic therapy of bone metastases with $153 \mathrm{Sm}$-EDTMP. Eur J Nucl Med Mol Imaging. 2014; 41: 238-252. Ref.: https://goo.gl//10A8m

4. Hamilton JG, Lawrence JH. Recent clinical developments in the therapeutic application of radiophosphorus and radio-iodine. J Clin Invest. 1942; 21: 624. Ref.: http://bit.ly/2XIR7h8

5. Lawrence $\mathrm{JH}$. Nuclear physics and therapy. Preliminary report on a new method for the treatment of leukemia and polycythemia vera. Radiology. 1940; 35: 51-60. Ref.: http://bit.ly/2ZqDaEZ 
6. Hertz S, Roberts A. Radioactive iodine in the study of thyroid physiology; the use of radioactive iodine therapy in hyperthyroidism. J Am Med Assoc. 1946; 131: 81-86. Ref.: https://goo.gl/mmYoKk

7. Goldenberg DM, Preston DF, Primus FJ, Hansen HJ. Photoscan localization of GW-39 tumors in hamsters using radiolabeled anticarcinoembryonic antigen immunoglobulin G. Cancer Res. 1974; 34: 1-9. Ref.: https://goo.gl/OmrVv1

8. Goldenberg DM, DeLand F, Kim E, Bennett S, Primus FJ, et al. Use of radiolabeled antibodies to carcinoembryonic antigen for the detection and localization of diverse cancers by external photoscanning. N Engl J Med. 1978; 298: 1384-1386 Ref.: https://goo.gl/Gk1VwV

9. Das T, Pillai MR. Options to meet the future global demand of radionuclides for radionuclide therapy Nucl Med and Biol. 2013; 40: 23-32. Ref.: https://goo.gl/EiuUuq

10. Lee DY, Li KC. Molecular theranostics: a primer for the imaging professional. AJR Am J Roentgenol. 2011; 197: 318-324. Ref.: https://goo.gl/0h8Zml

11. Pacilio M, Ventroni G, De Vincentis G, Cassano B, Pellegrini R, et al. Dosimetry of bone metastases in targeted radionuclide therapy with alpha-emitting (223)Ra-dichloride. Eur J Nucl Med Mol Imaging. 2016; 43: 21-33. Ref.: https://goo.gl/HVOEZP

12. Pacilio $M$, Ventroni $G$, Cassano $B$, lalongo $P$, Lorenzon $L$, et al. A case report of image-based dosimetry of bone metastases with Alpharadin ( (223 Ra)-dichloride) therapy: inter-fraction variability of absorbed dose and follow-up. Ann Nucl Med. 2016; 30: 163-168. Ref.: https://goo.gl/mcOeey

13. Baidoo KE, Yong K, Brechbiel MW. Molecular pathways: targeted a-particle radiation therapy. Clin Cancer Res. 2013; 19: 530-537.Ref.: https://goo.gl/XSRSgu

14. Wild D, Frischknecht $M$, Zhang $H$, Morgenstern A, Bruchertseifer $F$, et al. Alpha-versus beta-particle radiopeptide therapy in a human prostate cancer model (213Bi-DOTA-PESIN and 213Bi-AMBA versus 177Lu-DOTA-PESIN). Cancer Res. 2011; 71: 1009-1018. Ref.: https://goo.gl/zxLPpa

15. Lewington VJ. Bone-Seeking Radionuclides for Therapy. J Nucl Med. 2005; 46: 38S-47S. Ref.: https://goo.gl/fBQ3R7

16. Czernin J, Benz MR, Allen-Auerbach MS. PET/CT imaging: The incremental value of assessing the glucose metabolic phenotype and the structure of cancers in a single examination. Eur $\mathrm{J}$ Radiol. 2010; 73: 470-480. Ref.: https://goo.gl/zabXUf

17. Maecke HR, Reubi JC. Somatostatin Receptors as Targets for Nuclear Medicine Imaging and Radionuclide Treatment. J Nucl Med. 2011; 52: 841-844. Ref.: https://goo.gl/cz9aRd

18. Velikyan I. Molecular imaging and radiotherapy: Theranostics for Personalized Patient Management. Theranostics. 2012; 2: 424-426. Ref.: https://goo.gl/vzwt6U

19. Rösch F, Baum RP. Generator-based PET radiopharmaceuticals for molecular imaging of tumours: on the way to THERANOSTICS. Dalton Trans. 2011; 40: 6104-6111. Ref.: https://goo.gl/1KqHaf

20. Adams S, Baum RP, Hertel A, Wenish HJ, Staib-Sebler E, et al. Intraoperative Gamma Probe Detection of Neuroendocrine Tumors. J Nucl Med. 1998; 99: 1155-1160. Ref.: https://goo.gl/0uCF8F

21. Shi J, Liu TWB, Chen J, Green D, Jaffray D, et al. Transforming a Targeted Porphyrin Theranostic Agent into a PET Imaging Probe for Cancer. Theranostics. 2011; 1: 363-370. Ref.: https://goo.gl/Y9YP1L

22. Del Vecchio S, Zannetti A, Fonti R, Pace L, Salvatore M. Nuclear imaging in cancer theranostics. Q J Nucl Med Mol Imaging. 2007; 5: 152-63. Ref.: https://goo.gl/3wMB7R

23. Krenning EP, Bakker WH, Breeman WA, Koper JW, Kooij PP, et al. Localisation of endocrine-related tumours with radioiodinated analogue of somatostatin. Lancet. 1989; 1: 242-244. Ref.: https://goo.gl/COLOhA 